\title{
(2) OPEN ACCESS \\ Implementation of bowel ultrasound practice for the diagnosis and management of necrotising enterocolitis
}

\author{
Karen M Alexander $10,1,2$ Sherwin S Chan, ${ }^{2}$ Erin Opfer, ${ }^{2}$ Alain Cuna, ${ }^{3}$ Jason D Fraser, ${ }^{3}$ \\ Shazia Sharif, ${ }^{4}$ Minesh Khashu, ${ }^{5,6}$
}

${ }^{1}$ GME Radiology, University of Missouri Kansas City, Kansas City, Missouri, USA

2Department of Radiology, Children's Mercy Hospitals and Clinics, Kansas City, Missouri, USA

${ }^{3}$ Department of Pediatrics, Children's Mercy Hospitals and Clinics, Kansas City, Missouri, USA

${ }^{4}$ Department of Paediatric Surgery, Royal London Hospital, London, UK

${ }^{5}$ Neonatal Service, Poole Hospital NHS Foundation Trust, Poole, UK

${ }^{6}$ Bournemouth University, Poole, UK

\section{Correspondence to} Dr Minesh Khashu,

Neonatology, Poole Hospital NHS Foundation Trust, Poole UK: minesh.khashu@nhs.net

Received 10 October 2019 Revised 25 March 2020 Accepted 30 March 2020 Published Online First 12 May 2020
Check for updates

(c) Author(s) (or their employer(s)) 2021. Re-use permitted under CC BY-NC. No commercial re-use. See rights and permissions. Published by BMJ.

To cite: Alexander KM, Chan SS, Opfer E, et al. Arch Dis Child Fetal Neonatal Ed 2021;106:F96-F103.

\section{ABSTRACT}

Necrotising enterocolitis (NEC) is a serious inflammatory bowel disease of prematurity with potentially devastating complications and remains a leading cause of morbidity and mortality among premature infants. In recent years, there has been accumulating data regarding benefits of using bowel ultrasound (BUS) in the diagnosis and management of NEC. Despite this, adoption of robust BUS programmes into clinical practice has been slow. As BUS is a relatively new technique, many barriers to implementation exist, namely lack of education and training for sonographers and radiologists, low case volume and unfamiliarity by clinicians regarding how to use the information provided. The aim of this manuscript is to provide a framework and a roadmap for units to implement BUS in day-to-day practice for NEC diagnosis and management.

\section{INTRODUCTION}

Necrotising enterocolitis (NEC) is characterised by overwhelming inflammation of the bowel wall causing intestinal injury and necrosis. Severe cases can ultimately lead to bowel perforation, widespread inflammatory response syndrome, multiorgan dysfunction and death. ${ }^{1}{ }^{3}$ While NEC is a disease that may progress rapidly, earlier diagnosis has the potential to improve outcomes through timely management including transfer to a surgical centre and surgical intervention as and when required. The mortality rate is higher after perforation; thus, earlier detection of severely ischaemic or necrotic bowel loops, before perforation occurs, could improve the morbidity and mortality in NEC. ${ }^{245}$ Efforts to reduce the burden of NEC have been hindered in large part by the lack of advancements in NEC diagnosis. ${ }^{67}$ Clinical examination and laboratory tests are not specific for the disease; clinicians thus depend on abdominal radiographs (AXRs) to aid in the diagnosis of NEC. ${ }^{8}$ It is well known, however, that AXR has significant limitations in diagnosing NEC. ${ }^{29}$ Diagnosis of NEC can be made when pathognomonic signs such as portal venous gas (PVG) or pneumatosis intestinalis are present; however, the sensitivity of these signs is relatively low especially in less severe cases of NEC. ${ }^{10}$ The most sensitive finding of bowel wall dilation, present in greater than $90 \%$ of cases, is not specific to NEC. ${ }^{2}$ Additional common but nonspecific findings include air-fluid levels, bowel wall thickening and ascites. The combination of low

\section{What is already known on this topic?}

- Advantages of bowel ultrasound (BUS) over abdominal radiograph (AXR) in necrotising enterocolitis (NEC) include real-time assessment of the bowel, earlier diagnosis and earlier identification of ominous findings.

- Implementation of BUS in clinical practice is often hindered by lack of training and multidisciplinary approach and uncertainty regarding significance of findings.

\section{What this study adds?}

Technique and methods to assist with performing the ultrasound examination and examples of important findings to assist in image interpretation.

- Clear explanations of the clinical importance of the various findings on BUS for NEC.

- Provides a framework for practice to assist in the implementation of BUS practice in a setting where it is not yet commonplace.

sensitivity for the specific signs and low specificity for the sensitive signs often results in equivocal results by plain radiography alone. ${ }^{2} 91112$

In recent years, the addition of bowel ultrasound (BUS) to the diagnostic evaluation of NEC has been shown to add value. BUS is a non-invasive imaging modality that is readily available in most neonatal intensive care units. This has been well studied through multiple meta-analysis and narrative reviews. ${ }^{13}{ }^{214-16}$ Advantages of BUS over AXR include exclusion of rare but clinically overlapping differential diagnoses such as ruptured appendicitis or intussception, ${ }^{17}{ }^{18}$ real-time assessment of peristalsis, vascular perfusion, bowel wall thickening and abdominal fluid as well as increased or earlier identification of PVG and pneumatosis. ${ }^{19}{ }^{4}$ BUS has improved diagnostic accuracy as compared with AXR for NEC diagnosis. BUS can detect early signs of NEC (such as bowel wall thickening, decreases in bowel perfusion and peristalsis), which can then translate to earlier treatment before more advanced NEC develops. ${ }^{15} 2021$ BUS can also detect more advanced signs of NEC such as bowel wall thinning, absent bowel perfusion and absent peristalsis, 
Box 1 List of appropriate indications for bowel ultrasound (BUS)

Appropriate indications for BUS

- Earlier diagnosis of necrotising enterocolitis (NEC).

- Establishing the diagnosis of NEC when abdominal radiograph (AXR) is equivocal.

- AXR demonstrating a gasless abdomen.

- Evaluating for complications in known NEC.

- Evaluation for features suggestive of need for surgical intervention in the setting of clinical deterioration.

which suggest impending bowel perforation. This knowledge can help guide clinicians to pursue more aggressive treatment options such as surgery before marked clinical deterioration occurs. Conversely, although BUS cannot definitively rule out NEC, it can provide reassurance that no findings suggestive of NEC are present. This can be especially helpful in cases where AXR is equivocal. In such cases, unnecessary treatment with broad-spectrum antibiotics and prolonged parenteral nutrition with associated poor outcomes may be avoided. ${ }^{22} 23$

Barriers to widespread utilisation of BUS in clinical practice are many. Given its relatively new nature, many have received no formal training on the subject. Sonographers, even paediatric sonographers, who have experience with BUS have previously used it mainly to look for appendicitis or intussusception. Similarly, many radiologists have minimal experience in BUS outside of these indications and may be uncomfortable interpreting the images for NEC. Both of these pathologies are most often imaged in an age group much older than that of NEC, and therefore, the overlap is minimal. In addition, depending on the facility size, there may only be a handful of cases that would appropriately use BUS yearly, further limiting exposure to cases. Clinicians may be hesitant to order BUS, unsure of both the indications, as well as how findings should impact care. The goal of this paper is to provide clear methods and examples to assist with implementation of the examination, interpretation of images and clear explanations of the clinical importance of the various findings on BUS for NEC.

The appropriate uses of BUS in the evaluation of NEC are summarised in box 1 .

\section{PATHOLOGICAL CORRELATION WITH SONOGRAPHY}

Current understanding of the pathogenesis of NEC is that of disequilibrium between injury and repair of the intestinal mucosa, linked to various risk factors: genetic predisposition, impaired immunity, prematurity, enteral feeding, bacterial colonisation and intestinal ischaemia. ${ }^{721} 24$

Microscopically, early NEC is seen as intestinal inflammation and mucosal oedema. Later findings include necrosis of the mucosa and villus destruction, leading to complete mucosal loss, then to transmural necrosis with complete loss of epithelial and muscular architecture. ${ }^{25}$

The sonographic appearance of bowel has a corresponding predictable sequence of changes. Early injury of the bowel wall results in inflammation and bowel wall oedema seen on sonography as thickened hyperaemic bowel wall. This progresses to thinned bowel wall with decreased flow and decreased or absent peristalsis, and next to extreme bowel wall thinning with absent flow and absent peristalsis, corresponding with bowel death, and leading to eventual perforation. ${ }^{26}$ Intramural gas, or pneumatosis, is air within the bowel wall. It is an indication of injury to the mucosa and is most frequently seen as an intermediate or late finding. PVG is an extension of intramural gas into the portal venous system.

\section{BUS TECHNIQUE}

\section{Preparation}

BUS can be performed at any time. Importantly, infants do not need to maintain nothing by mouth (NPO) status prior to BUS evaluation. A nurse should be available during the exam to assist with positioning of lines/tubes and monitor and manage the baby in the event any clinical deterioration occurs.

\section{Equipment}

High frequency $(6-15 \mathrm{MHz})$ linear transducer for detail of bowel wall.

Low frequency $(2-9 \mathrm{MHz})$ curved transducer for evaluation of abdominal fluid/localised collection, and so on.

Box 2 Representative protocol for sonographer with key components of the exam

\section{Necrotising enterocolitis bowel ultrasound technique/} protocol

Scan all four quadrants

- RLQ $\rightarrow$ RUQ $\rightarrow$ LUQ $\rightarrow$ LLQ.

- Images in sagittal and transverse.

Greyscale

Bowel wall

- Thickness. Normal between $1 \mathrm{~mm}$ and 2-2.7 $\mathrm{mm}$.

- Echogenicity.

- Dilation.

- Peristalsis. Obtain cine clips. May have to watch for $>1 \mathrm{~min}$.

Pneumatosis

- If air is seen in bowel in supine position, change to decubitus to see if air shifts or remains in the wall.

Ascites

- Simple or complex.

Pneumoperitoneum

Colour Doppler

- Decreased pulse repetition frequency to see subtle hyperaemia or lack of flow.

- Portal vein: assess for portal venous gas.

- Superior mesentric artery and vein only if easily identifiable.

Spectral Doppler

- PVG=typical artefact sharp bidirectional spikes of Doppler

Tips shift superimposed on portal venous waveform.

- Ensure adequate pain control prior to exam, providing a dose of medication if needed.

- Patient does not need to be NPO.

- Greyscale.

- High frequency transducer for detail of wall.

Low frequency transducer to look for free fluid/abscess and so on.

- Harmonics=decreased artefacts and better resolution.

- Panoramic images can be obtained for long segment.

- Can do without compression, graded compression or graded anterior and posterior compression as patient condition permits. 
Table 1 A summary of statistical analysis of key BUS and AXR findings derived from previous meta-analyses, obtaining a range of data without further statistical analysis

\begin{tabular}{|c|c|c|c|c|c|c|}
\hline \multicolumn{7}{|c|}{ Statistical significance of key bowel ultrasound findings* } \\
\hline \multirow[b]{2}{*}{ BUS finding } & \multicolumn{5}{|c|}{ For the diagnosis of NEC } & \multirow{2}{*}{$\begin{array}{l}\text { Eventual need for } \\
\text { surgery or death } \\
\mathrm{OR}^{1516}\end{array}$} \\
\hline & Sens $(\%)^{28}$ & Spec $(\%)^{28}$ & AXR correlate & AXR Sens $(\%)^{10}$ & $\operatorname{AXR} \operatorname{Spec}(\%)^{10}$ & \\
\hline \multicolumn{7}{|l|}{ Early findings } \\
\hline Increased bowel perfusion & - & - & None. & - & - & NSS \\
\hline Simple (anechoic) ascites & 45 & 92 & $\begin{array}{l}\text { Bowel loops displaced centrally if } \\
\text { large volume. }\end{array}$ & - & - & NSS \\
\hline Dilated bowel & - & - & Dilated bowel. & - & - & $3.50-3.59$ \\
\hline \multicolumn{7}{|l|}{ Intermediate findings } \\
\hline Portal venous gas & 27 & 94 & PVG. & 13 & 100 & NSS \\
\hline Pneumatosis intestinalis & 48 & 91 & Pneumatosis. & 44 & 100 & $2.01-2.23$ \\
\hline Bowel wall thickening & 31 & 67 & Enlarged mucosal folds. & - & - & $3.7-4.74$ \\
\hline Increased bowel echogenicity & - & - & None. & - & - & 8.58 \\
\hline \multicolumn{7}{|l|}{ Late findings } \\
\hline Bowel wall thinning & 22 & 96 & None. & - & - & $7.11-7.97$ \\
\hline Absent peristalsis & 3 & 95 & $\begin{array}{l}\text { Stationary patulous bowel loops on } \\
\text { serial exams. }\end{array}$ & - & - & $8.19-10.68$ \\
\hline Absent bowel perfusion & - & - & None. & - & - & $6.08-6.99$ \\
\hline Focal fluid collections & 19 & 98 & None. & - & - & $15.37-17.92$ \\
\hline Complex (echogenic) ascites & - & - & None. & - & - & 11.28 \\
\hline Pneumoperitoneum & 27 & 94 & Pneumoperitoneum. & 52 & 92 & $8.25-9.63$ \\
\hline
\end{tabular}

*Data reported are a compilation of the currently known sensitivity and specificity as reported in the referenced meta-analyses. It should be noted that not all studies included reported data on all categories. Further research is needed in this area to elucidate the most accurate data.

AXR, abdominal radiograph; BUS, bowel ultrasound; NEC, necrotising enterocolitis; NSS, not statistically significant; PVG, portal venous gas.

\section{Technique}

A representative summary of the exam protocol with key components and reminders is provided in box 2 .

Begin with supine greyscale (anatomic) imaging. This can be performed without compression for those too unstable to tolerate compression or with graded anterior and posterior compression. Harmonics, an advanced ultrasound setting available on almost all machines, will reduce artefacts and increase signal-to-noise ratio and can be used to provide better resolution. Scan all four quadrants beginning with the right lower quadrant, most commonly involved, and proceed clockwise. Both sagittal and transverse images should be obtained.

Bowel should be assessed for echogenicity, wall thickness, peristalsis, perfusion and pneumatosis. Presence of air in the dependent bowel wall is sufficient for diagnosis of pneumatosis. If air is seen in or near the antidependent bowel wall, it should be reassessed after change of infant position to decubitus (if patient condition allows) to differentiate pneumatosis from intraluminal gas. Panoramic images (extended view) can be obtained to show long segments of bowel. Evaluation for peristalsis should include cine clips. The bowel should be watched for up to $1 \mathrm{~min}$ before an assessment of absent peristalsis is made.

Colour doppler (flow) imaging should be used to determine intestinal mural blood flow. Settings of velocity $2-7 \mathrm{~cm} / \mathrm{s}$, the lowest possible pulse repetition frequency that does not produce aliasing and the highest colour gain that does not cause flash artefact may be required to identify low flow. ${ }^{27}$ Comparison of perfusion of adjacent bowel loops is beneficial in difficult cases.

Assess the portal vein for patency, flow and for PVG using greyscale and spectral Doppler (vascular waveform) imaging.

Evaluate for free fluid, focal fluid collections, echogenic fluid and pneumoperitoneum.

\section{KEY FINDINGS}

An overview of key ultrasound findings and their previously characterised statistical significance are compiled and summarised in table 1 .

\section{Pneumoperitoneum}

Pneumoperitoneum (figure 1) is seen as echogenic foci outside the bowel lumen, sometimes seen as stacked echogenic lines. In a supine infant, this is most commonly seen along the anterior

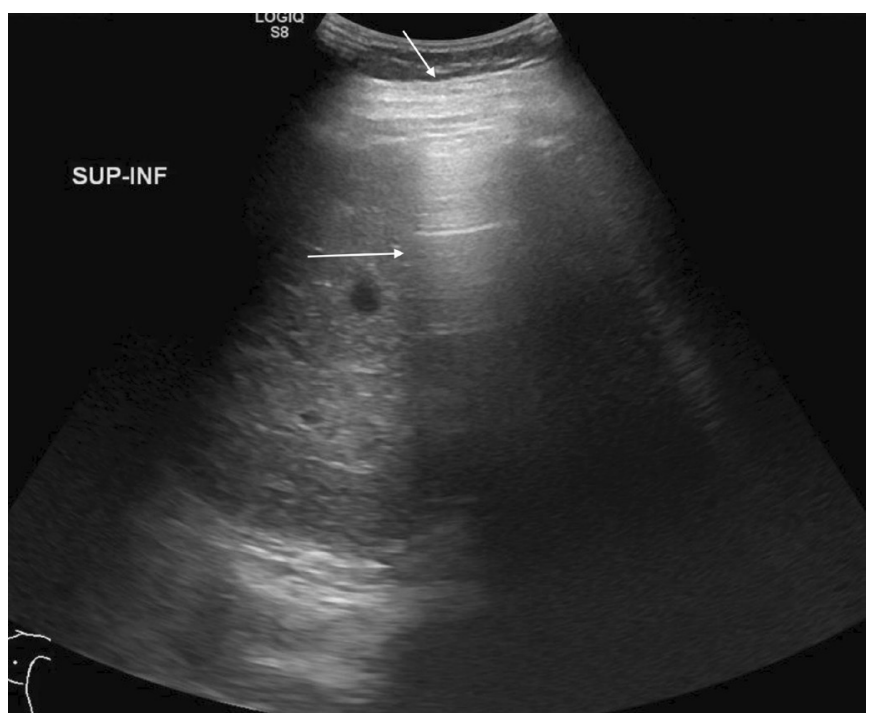

Figure 1 Hyperechoic foci with posterior reverberation artefact (arrows) just deep to the abdominal wall consistent with free intraperitoneal air. 


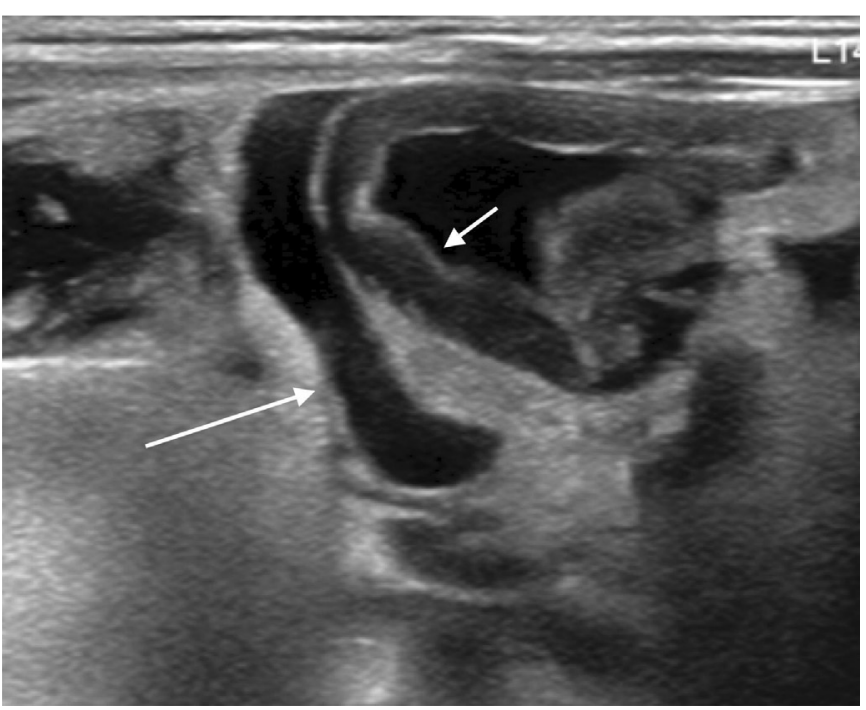

Figure 2 Layering debris within a collection of free fluid (long arrow) and adjacent loop of thickened bowel wall (short arrow).

abdominal wall. This is a nearly universal indicator for surgical consultation and intervention.

\section{Free fluid}

Intraperitoneal fluid should be evaluated as either simple or complex (figure 2). Simple free fluid is seen as anechoic regions surrounding intraperitoneal structures. Complex free fluid is echogenic, and loculations may be seen.

Free intraperitoneal fluid is often seen in NEC. However, small amounts of simple free fluid may be physiological in neonates. ${ }^{4}$ Large amounts of simple free fluid/ascites may be secondary to other conditions such as heart failure or renal failure and have a low sensitivity and specificity for NEC. ${ }^{28}$ In such instances, additional clinical and sonographic findings must be used to help determine the importance of this finding, and short-term follow-up BUS can be performed as necessary. Of note, amount of free fluid is a subjective determination based on the experience as there are currently no standards to differentiate 'large' amounts from 'small'.

Focal fluid collections (figure 3) or echogenic fluid (figure 4) are predictors of eventual need for surgery and are evidence of

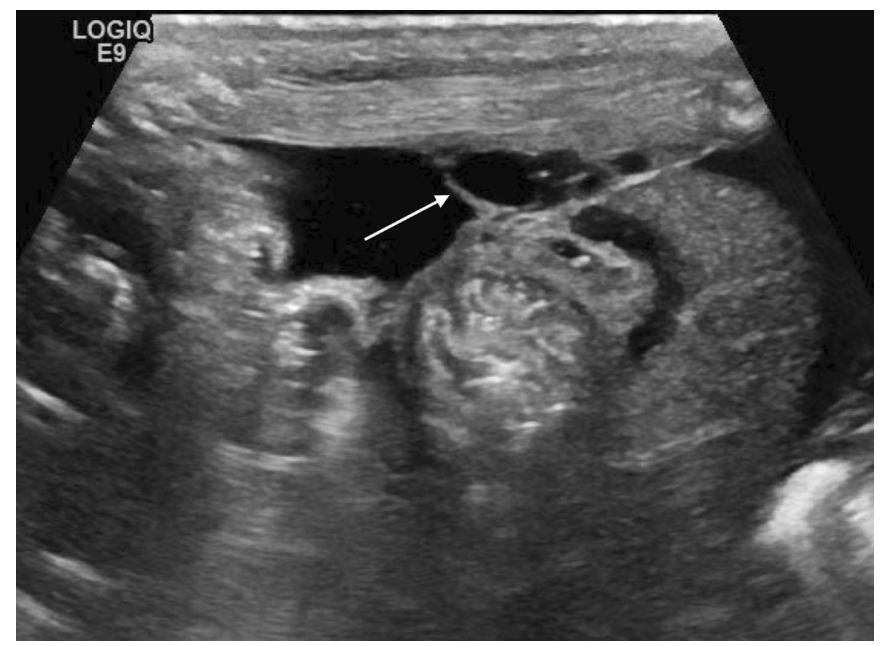

Figure 3 Focal fluid collection with septations (arrow) indicating bowel perforation.

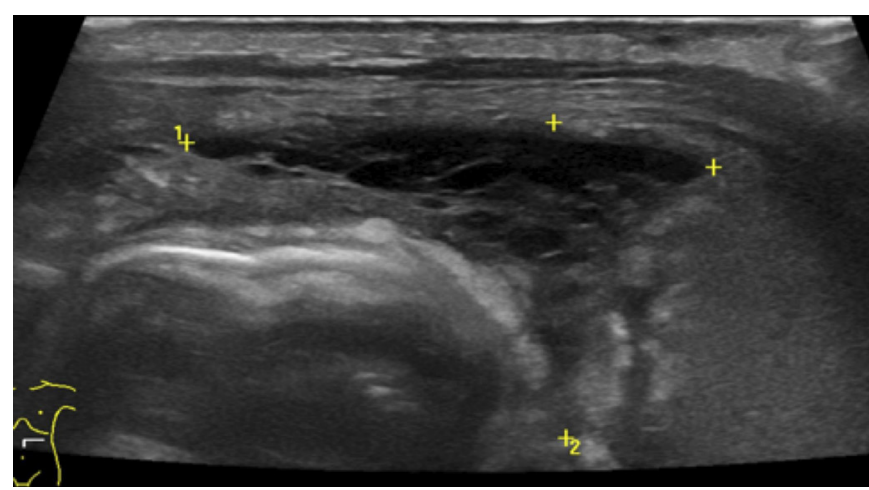

Figure 4 Echogenic fluid with septations (marked with calipers) adjacent to echogenic bowel.

perforation, even if pneumoperitoneum is not seen. ${ }^{1529}$ In these cases, a surgical consultation is warranted.

Pitfall: large amounts of free fluid may have a speckled appearance that can be confused for dense calcifications.

\section{Bowel wall}

Bowel should be evaluated as dilated or non-dilated. Bowel wall thickness should be graded as normal, thickened or thinned. Bowel wall measuring less than $1 \mathrm{~mm}$ can be considered thinned (figure 5). Cut-off value for thickening is less well established, with suggested values ranging from $2 \mathrm{~mm}$ to $2.7 \mathrm{~mm}$ (figure 2). ${ }^{14-16}$ Peristalsis should be noted as present or absent.

Bowel wall echogenicity should be assessed. The abdominal wall musculature can be used as a reference. The normal bowel wall (figure 6) should consist of five distinct layers (from outside to lumen): echogenic serosa, hypoechoic muscularis propria, echogenic submucosa, hypoechoic muscularis mucosa and echogenic interface between the gut lumen and the mucosa. ${ }^{30}$ Loss of clear visualisation of the hypoechoic muscularis layer should be termed increased bowel echogenicity (figure 4).

Bowel wall thickening, bowel wall thinning, increased wall echogenicity and loss of motility are associated with poor outcomes, while pneumatosis is associated with poor outcomes if in combination with other findings. ${ }^{15} 29$

Pitfall: intraluminal gas or intramural pneumatosis can mimic increased bowel wall echogenicity. These can be differentiated by posterior shadowing, which does not occur in bowel wall hyperechogenicity.

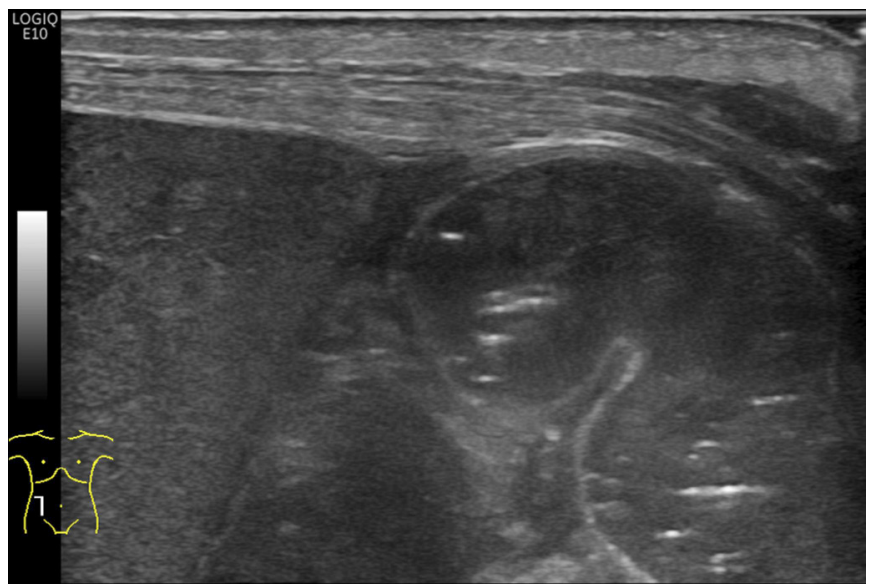

Figure 5 Loop of bowel with thinned wall adjacent to the liver. 


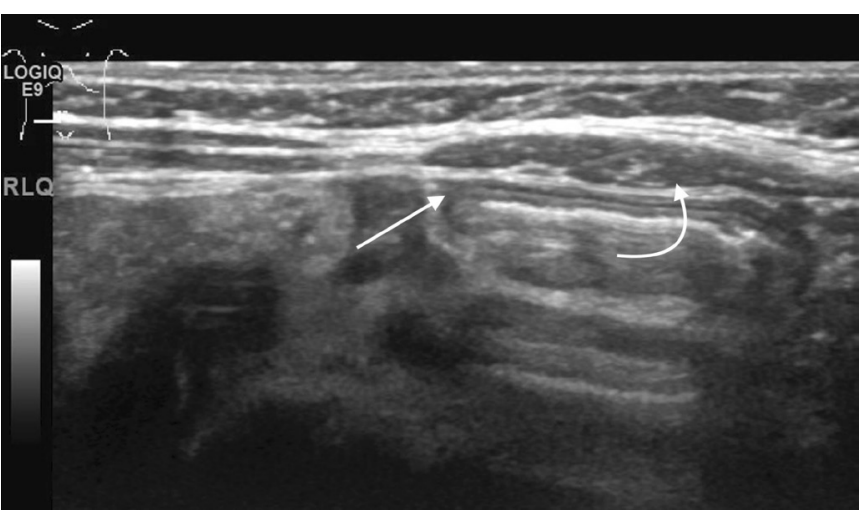

Figure 6 Normal thickness and echogenicity of the bowel wall (straight arrow) with adjacent abdominal wall musculature (curved arrow) as a comparison.

\section{Bowel wall perfusion}

Perfusion should be graded as normal, increased, decreased or absent. Normal perfusion can be considered between 1 and 9 colour dots per $\mathrm{cm}^{2}$ of bowel (figure 7). Patterns of hyperaemia include the 'zebra' pattern, the Y pattern (figure 8) and the circular pattern. ${ }^{42731}$

Reduced bowel wall perfusion is equivocal when in isolation but is associated with poor outcomes if in association with other findings. ${ }^{152829}$ Absent bowel wall perfusion (figure 9) is associated with poor outcomes.

\section{Pneumatosis}

Pneumatosis or intramural gas is seen as hyperechoic foci within the bowel wall (figures 10 and 11). It can vary from a single echogenic focus to circumferential bowel involvement. ${ }^{14}$

The presence of pneumatosis has been historically considered a pathognomonic sign of NEC and can be used to make the definitive diagnosis of NEC. However, as it may be a transient finding, its absence cannot rule out NEC. In addition, the presence of pneumatosis does not have a strong correlation with overall outcome within cohorts of infants with NEC. ${ }^{1528}$ Therefore, treatment in positive cases of pneumatosis should be determined by clinical findings as non-surgical management may be sufficient.

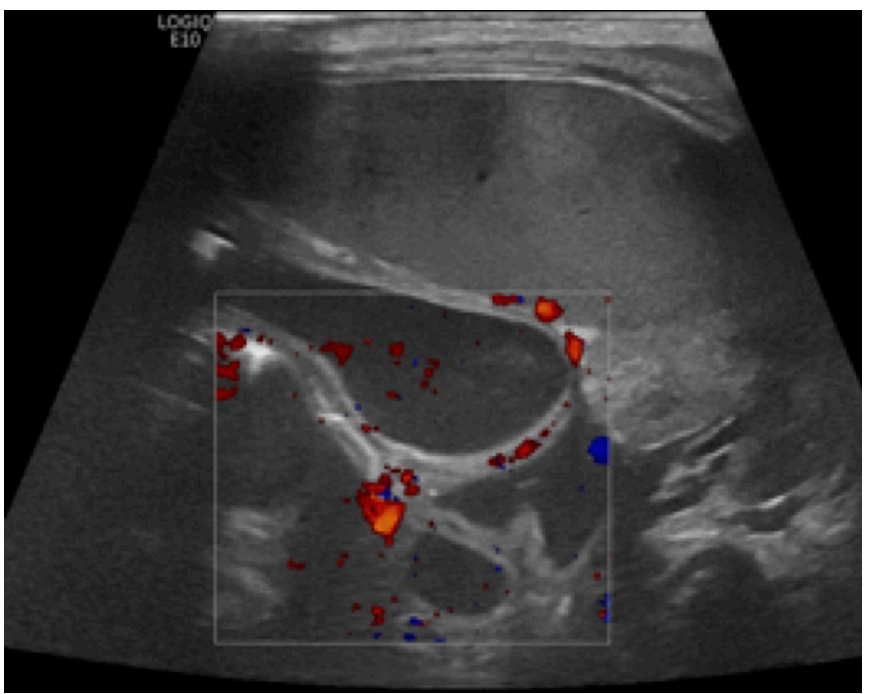

Figure 7 Thinned bowel wall with normal vascularity.

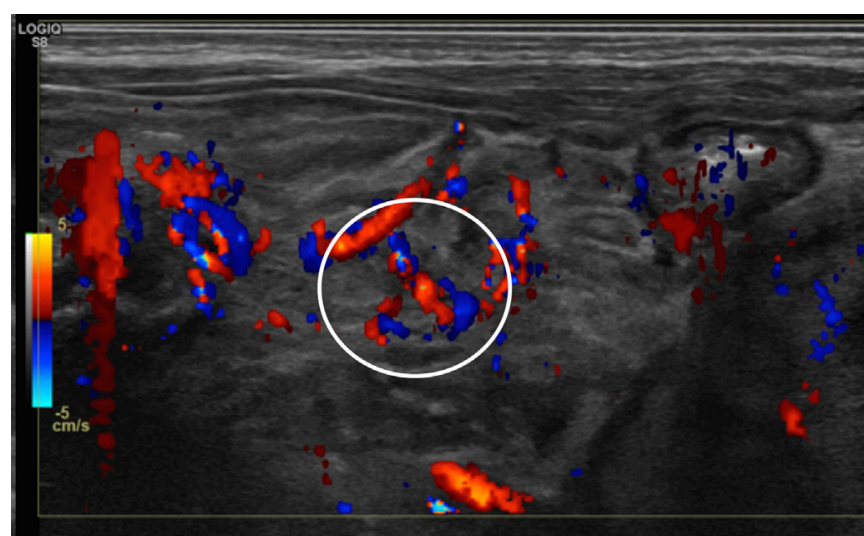

Figure 8 Bowel wall hyperaemia with the Y pattern seen (circle).

Pitfalls: small amounts of intramural gas may be misinterpreted as being intraluminal gas. Intraluminal gas may lie adjacent to the antidependent wall and be mistaken for pneumatosis. These can all be differentiated by imaging in both supine and decubitus positions. Gas that moves to remain antidependent is intraluminal, while intramural gas is confined within the bowel wall and therefore will not change position.

\section{Portal venous gas}

PVG is seen as echogenic foci within the portal venous system or in a branched pattern within the distal liver parenchyma, corresponding with portal venous branch vessels (figure 12). It may be seen moving distally within the central vessels on cine clips. On spectral Doppler waveforms, it is seen as sharp bidirectional spikes superimposed on the underlying waveform.

PVG is caused by intramural gas extending to the mesenteric venous system and passing into the portal venous system. As this is transient, it has a low sensitivity but high specificity for diagnosing definite NEC. The amount of PVG does not directly correlate with the amount of pneumatosis. Therefore, although presence can establish the diagnosis of NEC, it is of limited value in terms of clinical management beyond this. ${ }^{32}$ Specifically, PVG does not have a strong association with overall outcomes and has

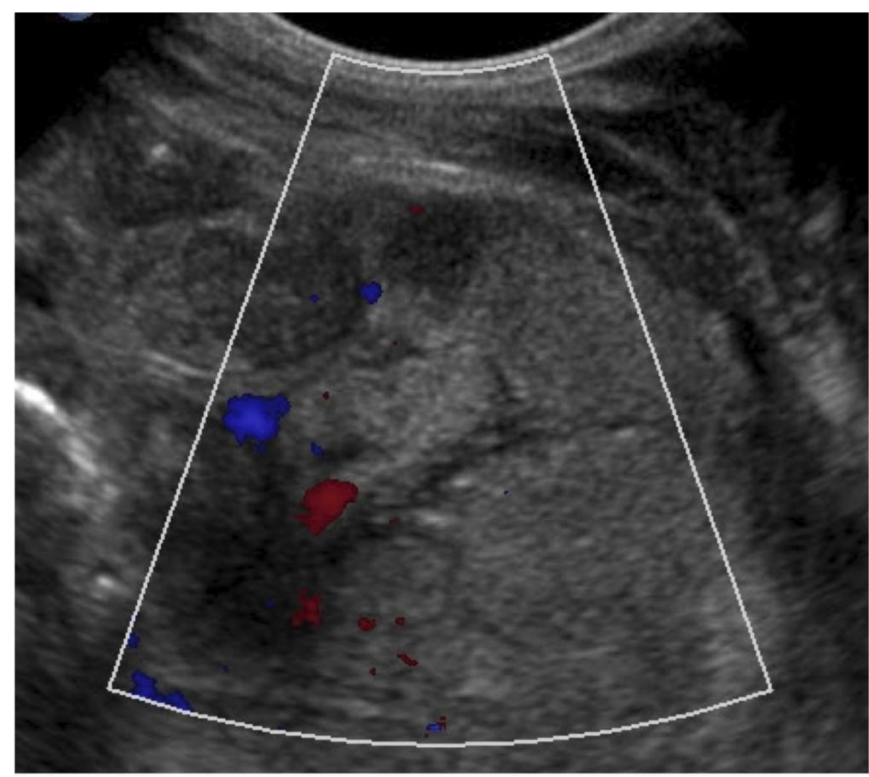

Figure 9 Several loops of bowel with areas of diminished and absent perfusion. The bowel wall is hyperechoic with indistinguishable layers. 


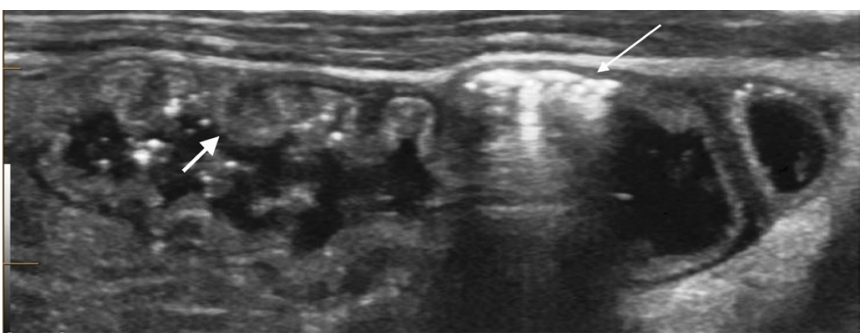

Figure 10 Hyperechoic foci with reverberation artefact within the bowel wall (thin arrow) consistent with pneumatosis. Adjacent thickening of the valvulae in an area of slightly hyperechoic bowel wall (thick arrow).

not been found to be a predictor of surgical necessity. ${ }^{1528}$ Treatment should be determined by clinical findings as non-surgical management may be sufficient.

Pitfalls: umbilical venous catheter manipulation can cause iatrogenic PVG; however, this can usually be determined based on history. Pneumobilia, or air within the bile ducts, can appear similar, however should be more centrally located than PVG and is uncommon in the neonatal period.

An overview for quick clinical reference of the key findings and their relative significance are summarised in box 3 .

\section{LIMITATIONS}

Like all ultrasound exams, BUS is operator dependent, and therefore variability in diagnostic accuracy is present. Additional inter-reader variability is present, particularly in distinguishing between intraluminal air and free air. These limitations can be mitigated or improved by training a select group of professionals (eg, sonographers, radiologists and neonatologists) at each institution and increasing the reproducibility of the exam at that institution. The pitfall of particular portions of the exam have been mentioned previously. Additionally, patient factors can limit the exam. Prominent artefact may be produced by ventilators and unstable infants may not tolerate the manipulation required to perform the full examination, limiting the utility in these subsets of infants. During image interpretation, we recommend that providers refer to table (pearls: most clinically significant findings) so that they can focus their attention on the findings most likely to change clinical management.

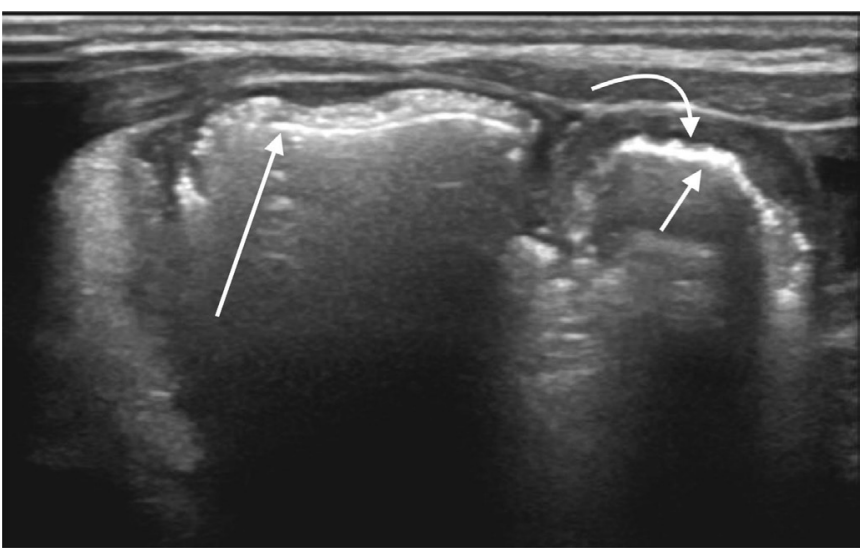

Figure 11 Small shadowing foci within the bowel wall consistent with pneumatosis (long arrow). Additional intraluminal air (short arrow) is seen in the adjacent segment of bowel, differentiated clearly from the bowel serosa (curved arrow).

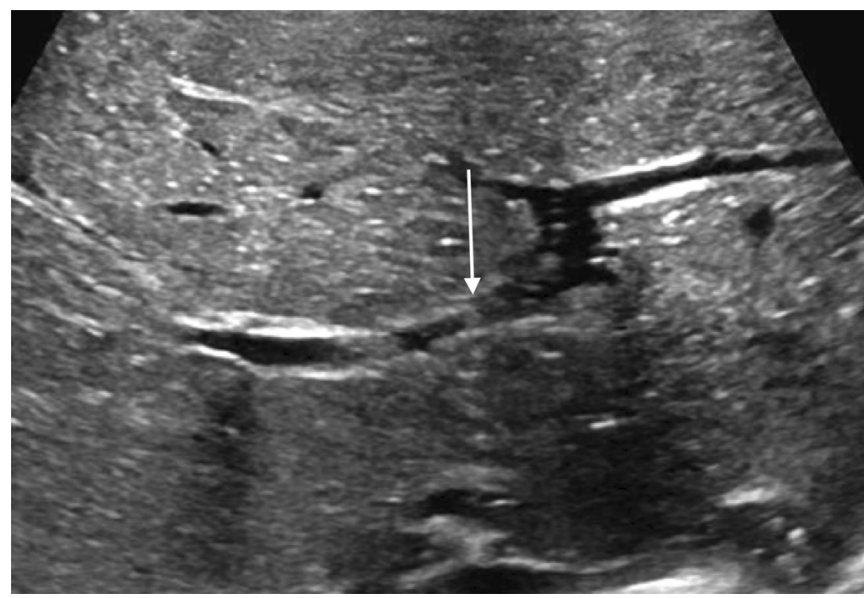

Figure 12 Shadowing hyperechoic foci within the right portal vein consistent with portal venous gas (arrows).

\section{CLINICAL QUESTIONS}

\section{How can BUS be used in the evaluation of NEC?}

BUS can be used as an adjunct to AXR to aid in diagnosis of infants with clinical suspicion of NEC by providing more detailed evaluation of the intestine. BUS can also be used for follow-up of infants with either confirmed NEC or continued suspicion for NEC to monitor for disease progression and guide clinicians regarding management.

In a preterm infant with concern for NEC, can a normal BUS alone be sufficient to rule out NEC?

No. Although BUS provides more information than AXR in the evaluation of NEC, its overall sensitivity and negative predictive value is still relatively low. Relying on a negative BUS alone can thus result in missing NEC. In a cohort of 100 infants with suspected NEC and a hypothetical overall prevalence of $\sim 50 \%$, Cuna et $a l^{28}$ estimated that as much as 25-40 infants with a negative BUS can actually have NEC (ie, false negatives). Thus,

\section{Box 3 Key findings and their relative clinical significance}

Pearls: most clinically significant findings

Findings with high specificity for need for surgery and increased mortality

- Focal fluid collections.

- Complex (echogenic) ascites.

- Pneumoperitoneum.

- Bowel wall thinning.

- Absent bowel peristalsis.

- Absent bowel wall perfusion.

- Increased bowel wall echogenicity.

Findings with high specificity for NEC diagnosis but not associated with need for surgery or increased mortality

- Pneumatosis intestinalis.

- Portal venous gas.

Findings not specific for necrotising enterocolitis diagnosis or need for surgery

- Increased bowel perfusion.

- Simple ascites.

- Bowel wall thickening. 
BUS should always be interpreted in conjunction with the overall clinical picture.

\section{In a preterm infant with suspicion for NEC but a non- diagnostic $A X R$, how reliable is a positive BUS in ruling in NEC?}

A meta-analysis ${ }^{28}$ of studies that used BUS for evaluation of infants with suspected NEC found that BUS has high specificity and positive predictive value for diagnosing NEC. Therefore, a non-diagnostic AXR alone should not negate a suspicious BUS. BUS findings most reliable for diagnosing NEC include PVG, pneumatosis intestinalis, free air, bowel wall thinning, absent peristalsis and focal fluid collection. Findings of extreme bowel wall thinning and absent perfusion/peristalsis suggest impending perforation. In contrast, bowel wall thickening and simple ascites-especially if found in isolation-are less reliable signs for NEC diagnosis. ${ }^{1528}$

\section{What is the role of serial BUS for follow-up of infants with confirmed NEC (Bell stage $\geq 2$ )?}

Serial BUS evaluation of infants with confirmed NEC can be used to monitor disease progression and response to treatment. Ominous signs concerning for impending bowel perforation include thinning bowel wall, absent bowel perfusion and absent peristalsis, while free air or focal fluid collections are often indicative that bowel perforation has already occurred. The presence of these BUS signs on follow-up supports the need for further escalation of care including surgery.

\section{Are there any concerns with the use of BUS for NEC?}

The major concern reported in the literature is clinical instability (ie, apnoea, bradycardia and/or desaturation events) during acquisition of BUS images. ${ }^{2}$ Continuous monitoring during procedure with frequent pauses for recovery as needed, as well as using large amounts of gel to limit abdominal pressure are usually sufficient for safe completion of BUS study. ${ }^{2733}$ BUS can also be limited when excessive amounts of overlying bowel gas is present. Theoretical concerns include: (1) overdiagnosis of NEC leading to an increase in unnecessary antibiotics and parenteral nutrition and (2) missed NEC leading to delayed treatment when infant actually has NEC. The relatively low sensitivity and high specificity of BUS for NEC suggests than missed NEC may be more likely than overdiagnosis.

\section{CONCLUSION}

Evidence regarding the benefits of BUS in the evaluation of NEC is growing, and clinicians are becoming increasingly aware of its advantages and potential availability. As BUS is quickly becoming an important tool in NEC imaging, it is imperative for radiologists to remain at the forefront of its implementation and interpretation and support its wider use on neonatal units. Implementation of BUS within the unit should include input from all aspects of the infants' care; a multidisciplinary team involving radiologists, surgeons, neonatologists, sonographers and nurses will help to ensure that best practices for a particular unit can be achieved. Initial implementation may be assisted by the use of a change package. Goals and measurable outcomes leading to success should be evaluated with the use of a specific methodology such as a driver diagram. Development of standard operating procedures to assist in clinical decision should be sought. Cases including imaging, treatments and outcomes should be reviewed regularly to ensure optimal performance and ongoing improvement. The techniques and examples laid out in this article are expected to provide foundational knowledge to help establish the use of BUS for NEC.

Contributors KMA: writing, submitting author and no disclosures. SSC: advising, editing, images and imaging knowledge. EO: writing, editing, images, imaging knowledge and advising. AC: neonatology clinical knowledge, clinical questions writing and editing. JDF and SS: paediatric surgery knowledge and editing. MK: corresponding author, conceptualised manuscript, advising, editing, neonatology clinical knowledge and insights.

Funding The authors have not declared a specific grant for this research from any funding agency in the public, commercial or not-for-profit sectors.

Competing interests SSC reports grants and personal fees from Jazz pharmaceuticals, outside the submitted work.

Patient consent for publication Not required.

Provenance and peer review Not commissioned; externally peer reviewed.

Data availability statement Data sharing is not applicable as no datasets are generated and/or analysed for this study. Not applicable.

Open access This is an open access article distributed in accordance with the Creative Commons Attribution Non Commercial (CC BY-NC 4.0) license, which permits others to distribute, remix, adapt, build upon this work non-commercially, and license their derivative works on different terms, provided the original work is properly cited, appropriate credit is given, any changes made indicated, and the use is non-commercial. See: http://creativecommons.org/licenses/by-nc/4.0/.

\section{ORCID iD}

Karen M Alexander http://orcid.org/0000-0001-5636-8339

\section{REFERENCES}

1 Neu J, Walker WA. Necrotizing enterocolitis. N Eng/ J Med 2011;364:255-64.

2 Epelman M, Daneman A, Navarro OM, et al. Necrotizing enterocolitis: review of state-of-the-art imaging findings with pathologic correlation. Radiographics 2007;27:285-305.

3 Patel RM, Kandefer S, Walsh MC, et al. Causes and timing of death in extremely premature infants from 2000 through 2011. N Engl J Med 2015;372:331-40.

4 Faingold R, Daneman A, Tomlinson G, et al. Necrotizing enterocolitis: assessment of bowel viability with color Doppler US. Radiology 2005;235:587-94.

5 Abdullah F, Zhang Y, Camp M, et al. Necrotizing enterocolitis in 20,822 infants: analysis of medical and surgical treatments. Clin Pediatr 2010;49:166-71.

6 Zani A, Pierro A. Necrotizing enterocolitis: controversies and challenges. F1000Res 2015;4:F1000:1373.

7 D'Angelo G, Impellizzeri P, Marseglia L, et al. Current status of laboratory and imaging diagnosis of neonatal necrotizing enterocolitis. Ital J Pediatr 2018;44:84.

8 Niño DF, Sodhi CP, Hackam DJ. Necrotizing enterocolitis: new insights into pathogenesis and mechanisms. Nat Rev Gastroenterol Hepatol 2016;13:590-600.

9 Buonomo C. The radiology of necrotizing enterocolitis. Radiol Clin North Am 1999:37:1187-98.

10 Tam AL, Camberos A, Applebaum H. Surgical decision making in necrotizing enterocolitis and focal intestinal perforation: predictive value of radiologic findings. J Pediatr Surg 2002;37:1688-91.

11 Kosloske AM, Musemeche CA, Ball WS, et al. Necrotizing enterocolitis: value of radiographic findings to predict outcome. AJR Am J Roentgenol 1988;151:771-4

12 Daneman A, Woodward S, de Silva M. The radiology of neonatal necrotizing enterocolitis (NEC). A review of 47 cases and the literature. Pediatr Radiol 1978;7:70-7.

13 Dilli D, Suna Oğuz S, Erol R, et al. Does abdominal sonography provide additional information over abdominal plain radiography for diagnosis of necrotizing enterocolitis in neonates? Pediatr Surg Int 2011;27:321-7.

14 Bohnhorst B. Usefulness of abdominal ultrasound in diagnosing necrotising enterocolitis. Arch Dis Child Fetal Neonatal Ed 2013;98:F445-50.

15 Cuna AC, Reddy N, Robinson AL, et al. Bowel ultrasound for predicting surgical management of necrotizing enterocolitis: a systematic review and meta-analysis. Pediatr Radiol 2018;48:658-66.

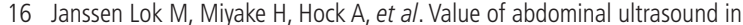
management of necrotizing enterocolitis: a systematic review and meta-analysis. Pediatr Surg Int 2018:34:589-612

17 Raveenthiran V. Neonatal appendicitis (Part 1): a review of 52 cases with abdominal manifestation. J Neonatal Surg 2015;4:4.

18 Martínez Biarge M, García-Alix A, Luisa del Hoyo M, et al. Intussusception in a preterm neonate; a very rare, major intestinal problem--systematic review of cases. $J$ Perinat Med 2004;32:190-4

19 Bohnhorst B, Kuebler JF, Rau G, et al. Portal venous gas detected by ultrasound differentiates surgical NEC from other acquired neonatal intestinal diseases. Eur $J$ Pediatr Surg 2011;21:12-17 
20 Garbi-Goutel A, Brévaut-Malaty V, Panuel M, et al. Prognostic value of abdominal sonography in necrotizing enterocolitis of premature infants born before 33 weeks gestational age. J Pediatr Surg 2014;49:508-13.

21 He Y, Zhong Y, Yu J, et al. Ultrasonography and radiography findings predicted the need for surgery in patients with necrotising enterocolitis without pneumoperitoneum. Acta Paediatr 2016;105:e151-5.

22 Cotten CM, Taylor S, Stoll B, et al. Prolonged duration of initial empirical antibiotic treatment is associated with increased rates of necrotizing enterocolitis and death for extremely low birth weight infants. Pediatrics 2009;123:58-66.

23 Stoll BJ, Hansen N, Fanaroff AA, et al. Late-Onset sepsis in very low birth weight neonates: the experience of the NICHD neonatal research network. Pediatrics 2002;110:285.

24 Choi YY. Necrotizing enterocolitis in newborns: update in pathophysiology and newly emerging therapeutic strategies. Korean J Pediatr 2014;57:505-13.

25 Tanner SM, Berryhill TF, Ellenburg JL, et al. Pathogenesis of necrotizing enterocolitis: modeling the innate immune response. Am J Pathol 2015;185:4-16.

26 Aliev MM, Dekhqonboev AA, Yuldashev RZ. Advantages of abdominal ultrasound in the management of infants with necrotizing enterocolitis. Pediatr Surg Int 2017:33:213-6.
27 Faingold R. Technical aspects of abdominal ultrasound and color Doppler assessment of bowel viability in necrotizing enterocolitis. Pediatr Radiol 2018;48:617-9.

28 Cuna AC, Lee JC, Robinson AL, et al. Bowel ultrasound for the diagnosis of necrotizing enterocolitis: a meta-analysis. Ultrasound Q 2018;34:113-8.

29 Ahle M, Ringertz HG, Rubesova E. The role of imaging in the management of necrotising enterocolitis: a multispecialist survey and a review of the literature. Eur Radiol 2018;28:3621-31.

30 Muradali D, Goldberg DR. US of gastrointestinal tract disease. Radiographics 2015;35:50-68.

31 Darge K, Anupindi S, Keener $\mathrm{H}$, et al. Ultrasound of the bowel in children: how we do it. Pediatr Radiol 2010;40:528-36.

32 Dördelmann M, Rau GA, Bartels D, et al. Evaluation of portal venous gas detected by ultrasound examination for diagnosis of necrotising enterocolitis. Arch Dis Child Fetal Neonatal Ed 2009;94:F183-7.

33 Staryszak J, Stopa J, Kucharska-Miąsik I, et al. Usefulness of ultrasound examinations in the diagnostics of necrotizing enterocolitis. Pol J Radiol 2015;80:1-9. 